\title{
Eddy Current- and Friction Brake Fusion by a Disc-Spring to a Hybrid-Brake
}

\author{
Christoph Holtmann, German Aerospace Centre and Christoph Köhler, German Aerospace Centre
}

\begin{abstract}
This paper shows the concept and design method of a hybrid brake. The magnetic attraction force between rotor and stator of an eddy current brake can be used to generate an additional mechanical friction torque. By using a disc spring between rotor and stator, the eddy current brake is extended to a so-called hybrid brake. In particular, the design method of the disc spring is the focus of this work. Using a system model that includes the electromagnetic and mechanical domains, the wear reduction compared to a conventional friction brake and the dynamic behavior depending on the spring parameters are investigated. Finally, a disc spring is designed in FEM with the desired force-displacement curve.
\end{abstract}

Index Terms - brake, eddy current brake, friction brake, retarder, disc-spring, diaphragm-spring, membrane-spring

\section{Introduction}

In the course of vehicle electrification, a large part of the braking energy to be converted can be recovered. However, a mechanical brake is still required for safety reasons. One reason is the need for a redundant system. Another reason is that the maximum required braking torque cannot be achieved with the electric drive system. One way to further reduce the use of a friction brake subject to wear is to use a wear-free eddy current brake. However, state-of-the-art eddy current brakes have the following disadvantages:

- very low power density compared to friction brakes

- braking to a standstill is not possible.

Figure I.1 shows the braking torques in a braking process with constant required torque $M_{\text {req }}$ and linearly decreasing speed $n$ when using an eddy current brake. At lower speeds, an additional mechanical friction brake must supply the difference between the required torque and the torque of the eddy current brake $M_{\mathrm{ec}}$ with torque $M_{\text {fric }}$.

In [1] it is shown that the disadvantage of low power density can be compensated by using a magnetoisotropic material structure. This structure allows to reduce the skin effect and to increase the free cooling area. The second disadvantage of the lack of torque at zero speed can be eliminated by extending the eddy current brake to a hybrid brake, as shown in patent [2]. Combinations of eddy current brakes and friction brakes are also presented in other publications. In [3], a concept is presented in which the eddy current brake and the friction brake are operated locally separately. In other concepts [4], [5], additional excitation poles are positioned on the

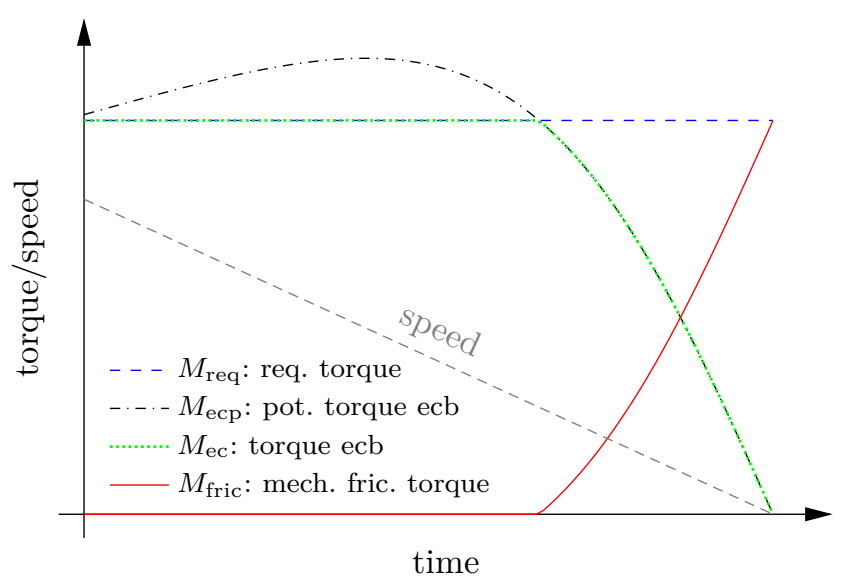

Fig. I.1: Theoretical torques of an eddy current brake with linearly decreasing speed over time and of a friction brake for low speeds.

brake disk to generate eddy currents and thus a wear-free portion of the braking torque. All these concepts generate the frictional torque via a classic brake caliper. However, the concept presented here uses the magnetic attraction between the rotor with exciter poles and the stator of an eddy current brake to generate the additional frictional torque. An additional actuator with power electronics or even a hydraulic system is therefore not required.

\section{Concept of the Hybrid Brake}

Figure II.2 shows an exemplary cad model and figure II.1 the superordinate operating principle of the hybrid brake. The current in the excitation windings on the rotor excites eddy currents in the stator, which generate an eddy current torque $M_{\mathrm{ec}}$ during the rotary motion.

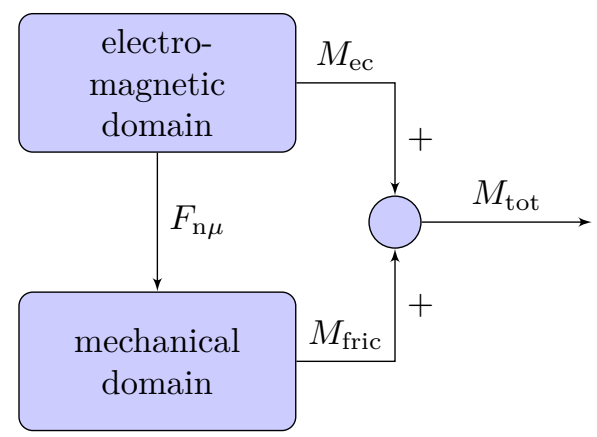

Fig. II.1: Superordinate operating principle of the hybrid brake 
At low speeds, the induction effect decreases sharply until, at zero speed, no braking torque is generated by eddy currents.

At these low speeds, the magnetic attraction force $F_{\mathrm{n} \mu}$ between the rotor and stator is used to generate a frictional torque $M_{\text {fric }}$ to brake to a stop. Since contact between the rotor and stator is necessary for the frictional action, a spring is used to ensure that contact is not present when the hybrid brake is not required to provide braking torque. Since the torque must be transmitted from the rotor to the hub and the rotor must be axially displaceable at the same time, a diaphragm spring is used.

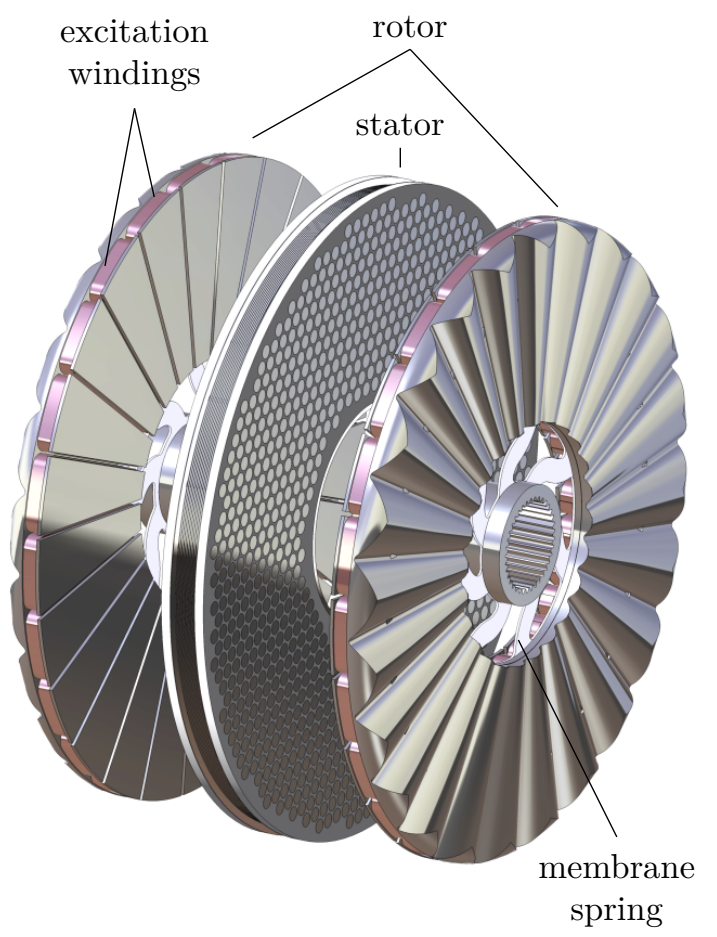

Fig. II.2: Exemplary CAD model of a hybrid brake

\section{Design Method}

Like the eddy current brake in [1] the hybrid brake is optimized for maximum wear reduction in the event of emergency braking compared with the use of a conventional friction brake. Since the wear of a mechanical friction brake is is approximately proportional to the converted braking energy [6], the wear reduction factor is defined as

$$
\triangle \varepsilon_{\mathrm{w}}=\frac{\int M_{\mathrm{ec}}(t) 2 \pi n(t) \mathrm{d} t}{\int M_{\mathrm{req}}(t) 2 \pi n(t) \mathrm{d} t}
$$

where $M_{e c}$ is the wear-free torque due to eddy currents and $M_{\text {req }}$ is the total required torque (see I.1). In the first step, the active magnetic geometry is optimized exactly according to the method in [1], neglecting the additional frictional torque. At best, a hybrid brake with this geometry can achieve the wear reduction factor from the optimization in which the frictional torque was neglected. The friction torque $M_{\text {fric }}$ of the hybrid brake results from the normal force $F_{\mathrm{ns}}$ acting on the surface with the mean radius $r_{\mathrm{m}}$ and the corresponding friction coefficient $\mu_{\text {fric }}$.

$$
M_{\text {fric }}=2 F_{\mathrm{ns}} r_{\mathrm{m}} \mu_{\mathrm{fric}}
$$

Using a spring with a stiffness close to zero, the normal force between the surfaces $F_{\mathrm{ns}}$ corresponds to the magnetic normal force $F_{\mathrm{n} \mu}$ between rotor and stator. To give a direct example, the torques in this case look like figure III.1. In this case, the maximum excitation current results in a normal force and thus a frictional torque which is represented by the red dashed line. Therefore, the excitation current must be reduced until the total torque is equal to the required torque. This means that the maximum excitation current must also be reduced at speeds where the torque due to eddy currents is lower than the required torque. As a result, the torque of the eddy currents is also greatly reduced and the wear reduction factor of the previous optimization cannot be achieved. Ideally, the excitation current should be set so

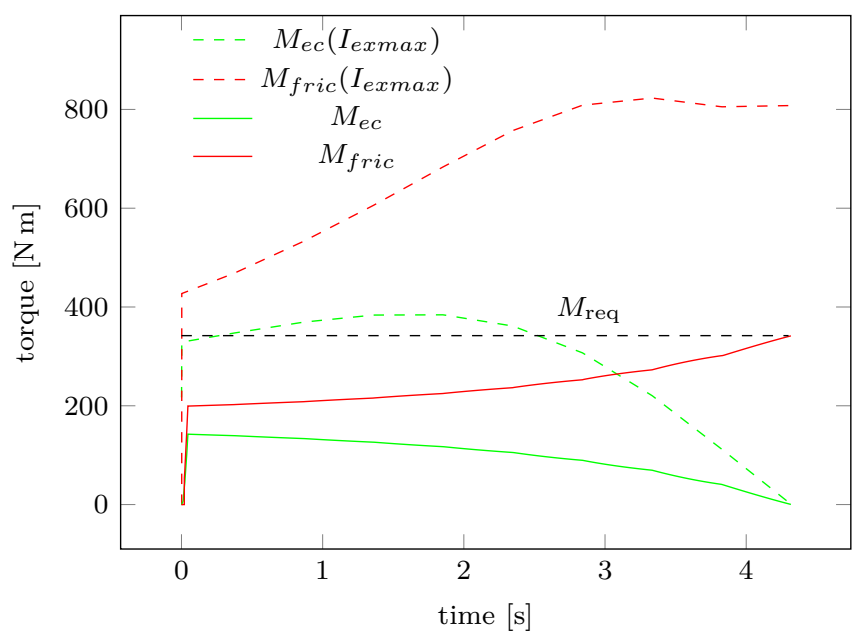

Fig. III.1: Torques over time for the use of a membrane spring with zero stiffness.

that the torque of the eddy currents, taking into account the boundary conditions $M_{\mathrm{ec}} \leq M_{\mathrm{req}}$ and $I_{\mathrm{ex}} \leq I_{\mathrm{exmax}}$ is as high as possible (see figure I.1). Thus, the spring mechanism must be designed to reduce the normal force $F_{\text {ns }}$ so that the wear reduction is as close as possible to the value of the previous optimization. In detail, this means that the spring characteristic should be optimized so that the time until the rotor contacts the stator $t_{\text {cont }}$ is as long as possible, so that the frictional torque is zero in this time range, which is the first quality factor for the spring parameter analyses. For stability reasons, the rotors should behave stably at a constant required torque. This means that the axial velocity should be mainly in 
one direction, i.e. as aperiodic as possible. An aperiodic factor is defined as a second quality parameter with

$$
\gamma_{\mathrm{ap}}=\frac{\int_{0}^{t=t_{\mathrm{con}}} \dot{s} \mathrm{~d} t}{\int_{0}^{t=t_{\mathrm{con}}}|\dot{s}| \mathrm{d} t}
$$

where $\dot{s}$ is the velocity of the rotor or the first derivative of the rotor position $s$ in figure IV.2. The aperiodic factor is one when the rotor moves in only one direction, and goes to zero when it oscillates back and forth for a long time. Since a high impact velocity of the rotors on the stator would result in a high peak normal force and thus a high peak mechanical torque, which is probably higher than the required torque, the impact velocity should be as low as possible. To provide an additional degree of freedom to meet these requirements, the hub of the rotor has a contour against which the spring can rest during compression. This contour can be used to influence the characteristics of the spring curve. The geometry of the spring is shown in figure V.2. The given parameters for the optimization are the allowable design space for the spring, the outer radius $r_{\text {spro }}$, its inner radius $r_{\text {spri }}$, its total thickness $h_{\text {spr }}$ and the allowable mechanical stress.

\section{System Model}

Figure IV.1 shows the block diagram of the hybrid brake system model. With the input voltage $u_{\mathrm{ex}}$, the excitation current in the excitation windings is calculated as a function of time $t$ with

$$
I_{\mathrm{ex}}(t)=\frac{1}{L_{\mathrm{ex}}} \int_{\tau=0}^{t}\left(u_{\mathrm{ex}}(t)-R_{\mathrm{ex}} I_{\mathrm{ex}}(t)\right) \mathrm{d} \tau
$$

where $R_{\mathrm{ex}}$ is the resistance and $L_{\mathrm{ex}}$ is the inductance of the excitation windings. The required input voltage $u_{\text {ex }}(t)$ is calculated in a model predictive control loop, which is not part of this work. The torque due to eddy currents $M_{\mathrm{ec}}$ as well as the magnetic normal force $F_{\mathrm{n} \mu}$ due to field coupling is described in lookup tables as a function of the excitation current $I_{\mathrm{ex}}$, the speed $n$ and the magnetic air gap $\delta_{\mu}$. For this purpose, the electromagnetic model of [7], which is extended by a co-energetic approach [8]-[10] to calculate the normal force, is used.

In figure IV.2 the model of the mechanical domain of the dynamic model is shown. The equation of motion of the rotor with the mass $m_{\mathrm{r}}$ is

$$
\frac{\mathrm{d}^{2} s}{\mathrm{~d} t^{2}}=\frac{1}{m_{\mathrm{r}}}\left(F_{\mathrm{n} \mu}+F_{\mathrm{l}}-F_{\mathrm{ls}}-F_{\mathrm{spr}}\right)
$$

where the forces of the stop limits are a combination of a elastic force expressed with a stiffness $c$ and damping force.

$$
F_{1}=\triangle s_{\text {is }} c_{l}+\frac{a \dot{s}}{\left(|\delta|+\delta_{0}\right)^{3}}
$$

In reality, the elastic force is the result of the deformation of the stator and the rotor. However, the deformation is represented by the overlap $\triangle s_{\text {is }}$ of solids. In the case of the stop against the stator, the intersection can be expressed by

$$
\triangle s_{\mathrm{is}}=\frac{\left|\delta_{\max }-s+s_{0}\right|-\left(\delta_{\max }-s+s_{0}\right)}{2}
$$

as it can be seen in the figure IV.2. It is always $\triangle s_{\text {is }} \geq 0$. The second term in equation (6) is the damping force. The damping force is a mixture of the damping due to a squeezed flow, when the air gap becomes very small, and the damping due to the internal friction of the bodies. The first damping phenomenon is described in [11]. With respect to the internal friction, the damping is inherently modelled with the squeezed damping equation by modifying equation 1 in [11] and choosing the parameters $a$ and $\delta_{0}$ appropriately.

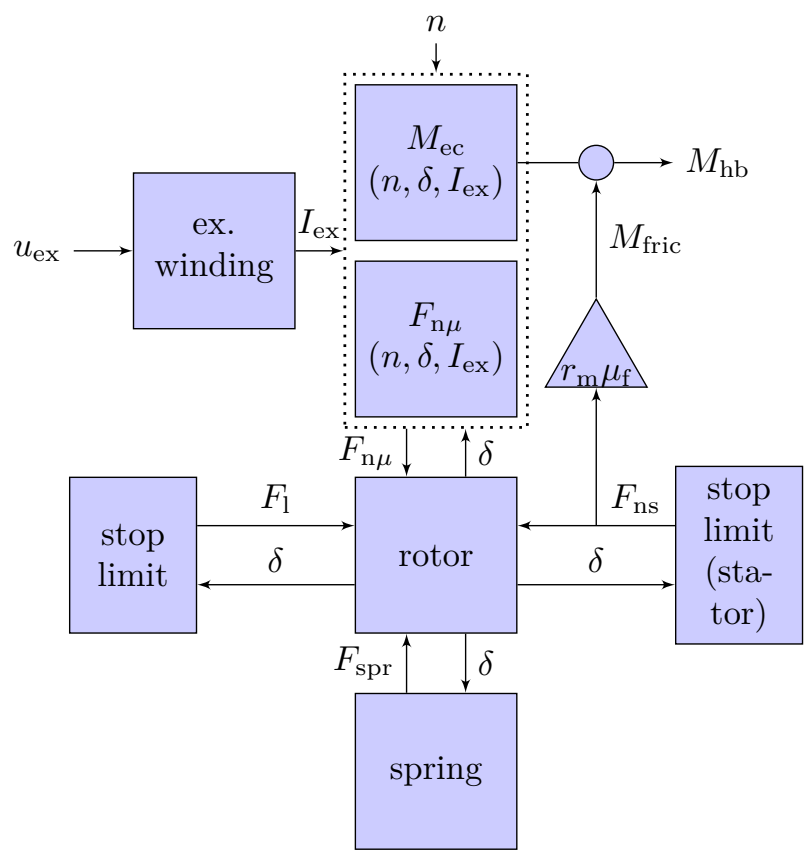

Fig. IV.1: System model of the hybrid brake

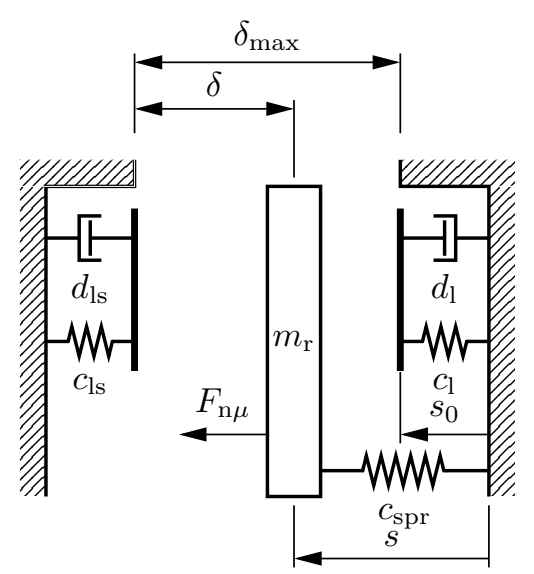

Fig. IV.2: Mechanical domain of the dynamic model 


\section{Spring Model}

The first approach to the spring is to design it as a wave spring. The spring will lay against a tangential contour during compression, as shown in the figure V.1. Due to the limited design space, the mean spring diameter $d_{\mathrm{ms}}$, the width $b_{\mathrm{s}}$ and the total height of the spring $h_{\text {stot }}$ are given. The parameters to be optimized are the spring wavenumber $N_{\mathrm{sw}}$, the thickness of a spring leaf $h_{\mathrm{ssh}}$ and the contour radius $r_{\mathrm{c}}$. The model is based

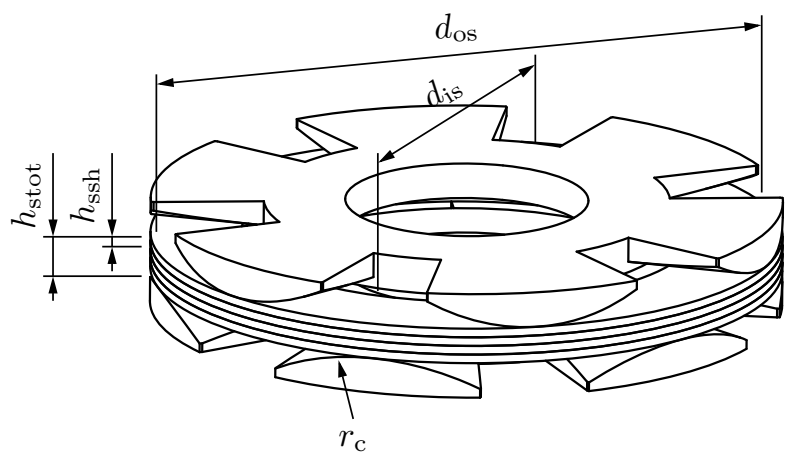

Fig. V.1: Schematic 3d geometry of the spring mechanism with non-deflected spring with a spring wave number of $N_{\mathrm{sw}}=6$.

on the Eulerian beam theory with the governing equation for the deflection $w$ along the path $x$

$$
E I \frac{\mathrm{d}^{4} w}{\mathrm{~d} x^{4}}=q
$$

Where $E$ is the Young's modulus, $I$ is the second moment of area, and $q$ is the specific surface load, which is zero in this case. Due to symmetry, a spring leaf is divided over $4 N_{\mathrm{sw}}$ quarter sections of length

$$
L_{\mathrm{qs}}=\frac{\pi\left(d_{\mathrm{os}}+d_{\mathrm{is}}\right)}{8 N_{\mathrm{sw}}}
$$

as shown in the figure V.2. Further the model is related to the middle spring sheet. When the spring comes into contact with the contour, the bending moment remains constant up to the end point of the contact due to the radius of curvature $r_{\mathrm{c}}+h_{\text {stot }} / 2$.

$$
M_{\mathrm{et}}=\frac{E I}{r_{\mathrm{c}}+\frac{h_{\mathrm{stot}}}{2}}
$$

If the force $F$ is given, the point at which the contact ends can be determined with

$$
x_{\mathrm{ec}}=L_{\mathrm{qs}}-\frac{M_{\mathrm{et}} 2 N_{\mathrm{sw}} N_{\mathrm{s}}}{F}
$$

Due to the strong deflection along the contour, the deflection of the free beam length is calculated in a separate coordinate system that is rotated by the angle

$$
\varphi=\arcsin \left(\frac{x_{\mathrm{ec}}}{r_{\mathrm{c}}+\frac{h_{\mathrm{stot}}}{2}}\right)
$$

and shifted by the vector

$$
x_{\mathrm{ec}} ; \quad y_{\mathrm{et}}=\sqrt{\left(r_{\mathrm{c}}+\frac{h_{\mathrm{stot}}}{2}\right)^{2}-x_{\mathrm{et}}^{2}}-r_{\mathrm{c}}
$$

with respect to the main coordinate system. The force $F$ acts with its component

$$
F_{\mathrm{fy}}=F \cos (\varphi)
$$

in the coordinate system on the free bar of length

$$
L_{\mathrm{f}}=L_{\mathrm{qs}}-\varphi r_{\mathrm{c}}
$$

and leads to a free deflection of

$$
s_{\mathrm{fy}}=\frac{F_{\mathrm{fy}} L_{\mathrm{f}}^{3}}{3 E I 2 N_{\mathrm{sw}} N_{\mathrm{s}}}
$$

The reverse transformation to the main coordinate system then leads to the deflection of the spring

$$
s=2\left\{s_{\mathrm{sy}} \cos (\varphi)+r_{\mathrm{c}}(1-\cos (\varphi))+L_{\mathrm{f}} \sin (\varphi)\right\}
$$

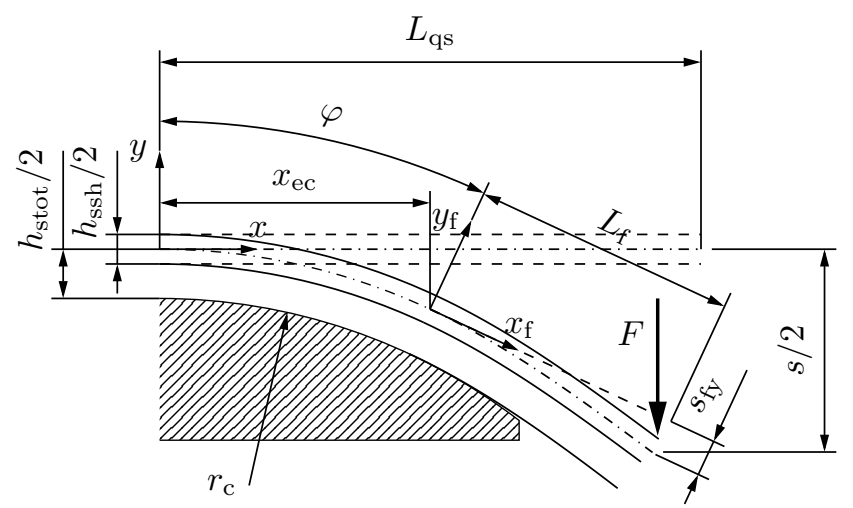

Fig. V.2: Quarter-Geometry of the middle, by the force $F$ deformed, spring sheet with the limiting contour

\section{Spring Parameter Analysis}

After a transient simulation of the system model, the quality criteria are evaluated by varying the spring parameters, each of which leads to different spring characteristics. Figure VI.1 shows different design points in the space of quality criteria. For a better understanding of the system, the behavoir is analysed in detail for three different design points. The red, green and blue design points (design 1-3) are each the result of spring parameters that produce the spring characteristics in figure VI.2. Each of these spring characteristics results in a different dynamic behavior, illustrated by the state trajectories in figure VI.3. It is evident that design 2 with the green spring curve results in the lowest time to rotor contact with the stator and the highest impact velocity because it has the lowest stiffness and preload. Design 1 with the red spring curve gives the highest time to impact, a very low impact velocity, but also a dynamic behavior with a high number of periodic oscillations (see figure VI.3). At some point, the spring force exceeds the 


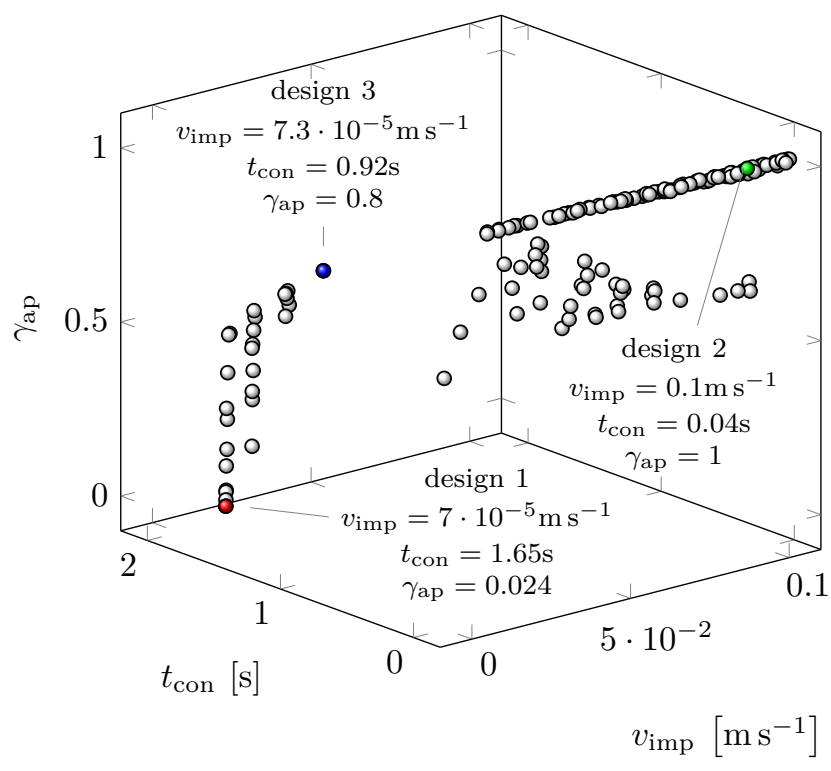

Fig. VI.1: Result of the spring parameter analyses. $t_{\text {con }}$ : time until contact, $v_{\text {imp }}$ : impact velocity, $\gamma_{\mathrm{ap}}$ : aperiodic factor. The coloured points (design 1 to 3 ) are related to the state space trajectories in figure VI.3.

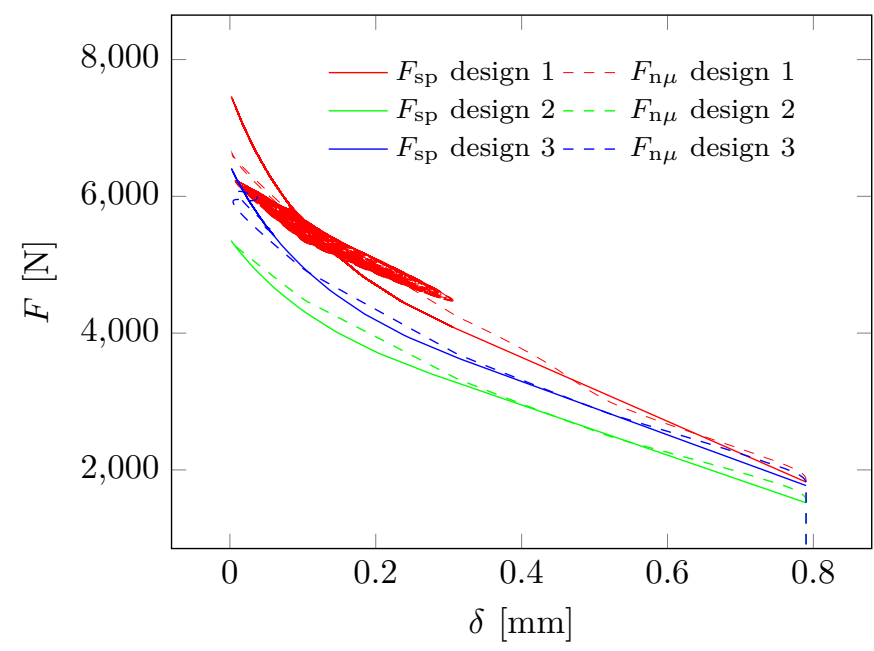

Fig. VI.2: Spring curves for spring parameters resulting in the quality criteria market with the red, green and blue point in figure VI.1 and corresponding time dependent magnetic normal forces projected in the spring curve plane.

magnetic attraction force, slowing the axial motion of the rotor and causing it to move back. Design 3 offers a tradeoff between a lower time to impact and a lower oscillation rate. In figure VI.4, it is clear that designs that result in a higher time to contact also have a higher wear reduction factor because the frictional torque stays at zero longer. Design 1 has the highest wear reduction factor of $\triangle \varepsilon_{\mathrm{w}}=0.9$, but also results in torque oscillations due to the unstable motion of the rotor. Design 3 is the design with the next highest wear reduction factor of $\Delta \varepsilon_{\mathrm{w}}=0.85$ and an acceptable aperiodic factor of $\gamma_{\mathrm{ap}}=0.8$. This design, which yields the blue spring curve, will be selected to build a functional demonstrator for future experiments. To ensure that the spring design will result in the desired spring curve in reality, an FEM analysis is performed.

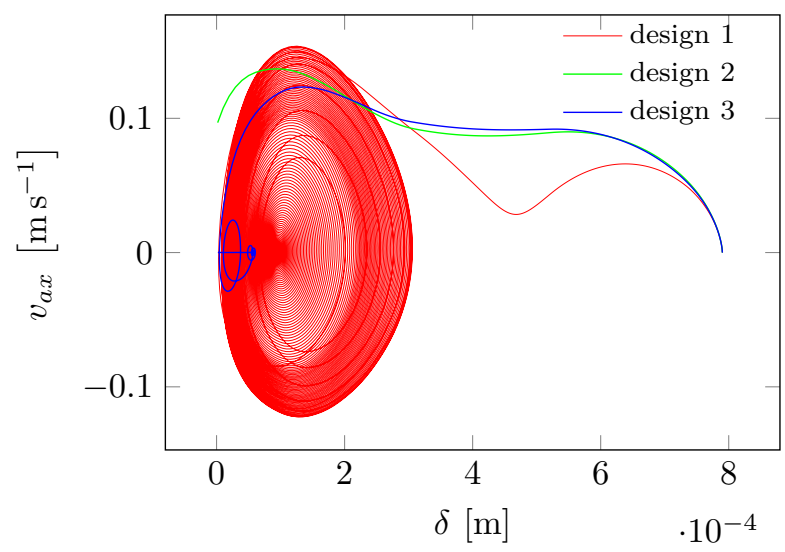

Fig. VI.3: State space trajectories for the axial movement of the rotor for different spring parameters resulting in different impact velocities, times to impact and aperiodic factors

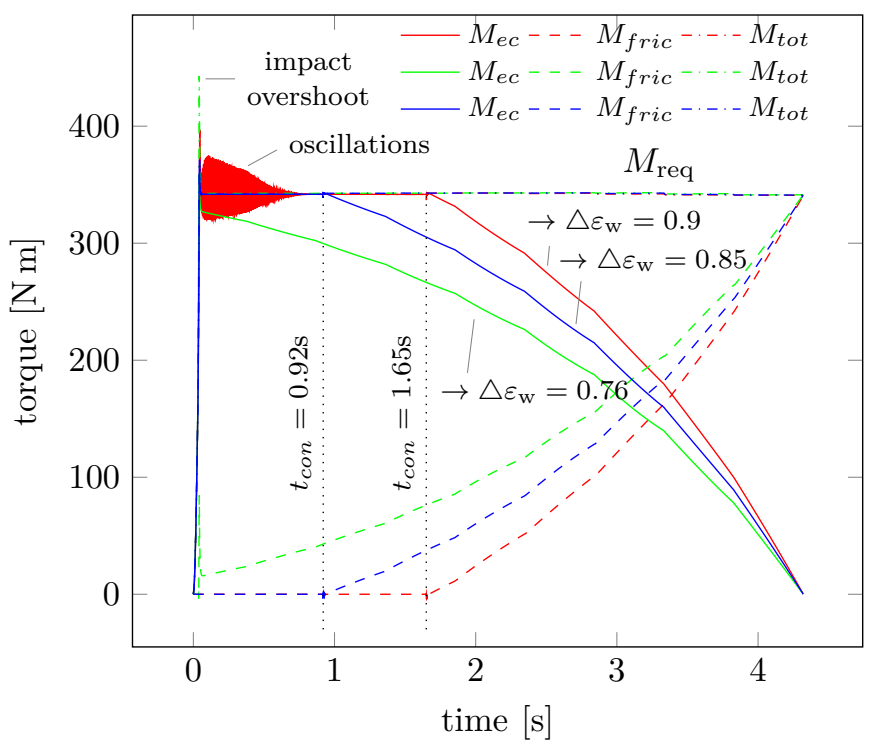

Fig. VI.4: Torques over time for the spring designs with the spring curves in figure VI.2

\section{FEM-Analyses}

During the design process, it was determined that the tangential contour spring mechanism would most likely be difficult to manufacture. The final design is axisymmetric and therefore much easier to manufacture. It consists of a diaphragm spring that can contact a radial contour of the hub. This design was not initially considered because it was thought that such a design would have too high a mechanical stress for the required forces for the given installation space. The stresses are high, with a value of $\sigma=1200 \mathrm{~N} \mathrm{~mm}^{-2}$, but a spring 
steel with a tensile strength of $\sigma_{\text {all }}=1800 \mathrm{~N} \mathrm{~mm}^{-2}$ is used. In order to achieve the desired spring characteristics even with the modified design, a parameter study is performed using the FEM model. Figure VII.1 shows an example of the relative mean square deviation of the spring characteristic from the required one, which is obtained for different contour radii $r_{\mathrm{c}}$. Figure VII.2 shows the spring characteristic curve of the final spring design calculated with fem. The relative mean square error of this spring curve is less than $3 \%$ to the desired one.

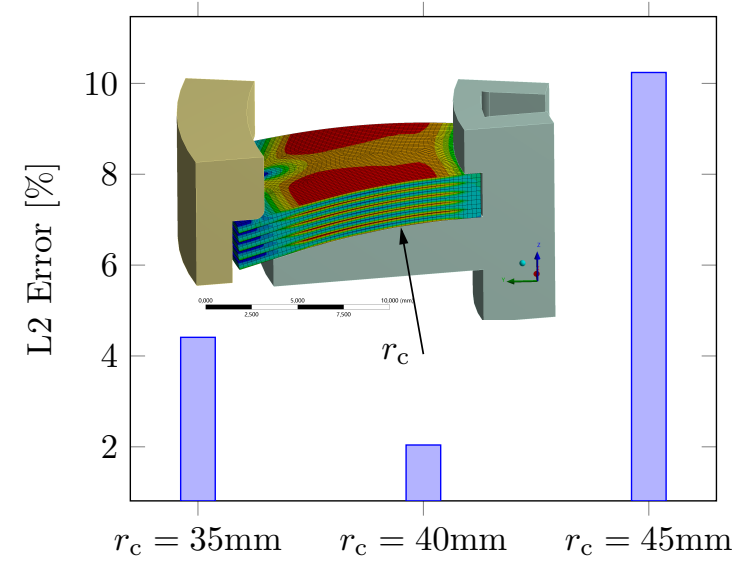

Fig. VII.1: Relative middle square error of spring curves to the desired for different contour radii $r_{\mathrm{c}}$.

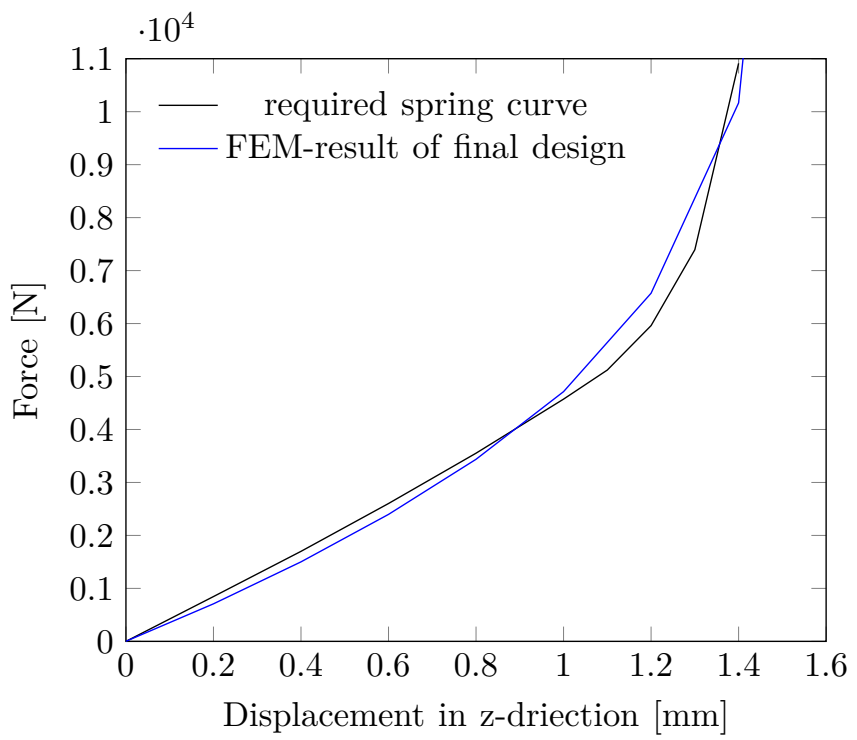

Fig. VII.2: Force-Displacement Curves of a membrane spring stack with five sheets

\section{Conclusion}

It is shown that the extension of an eddy current brake to a hybrid brake, which uses the magnetic attraction force between rotor and stator, is a feasible solution for braking to a standstill. However, the net attractive force must be reduced so that the mechanical frictional torque is as low as possible to maximize wear reduction. To achieve this, the spring mechanism that counteracts the magnetic attraction force is designed to maximize the time it takes for the rotor to contact the stator. This requirement can be achieved with an appropriate stiffness of the spring. However, it also turns out that a spring with a stiffness that leads to a maximum time to contact also results in a dynamic behaviour with strong oscillations. On the other hand, a spring with a stiffness that is too low results in a very low time to contact and thus a low wear reduction, but also in a high impact velocity. Due to the momentum of the axial movement, this causes the braking torque to overshoot. Ultimately, the spring curve that gives the next highest wear reduction of $\triangle \varepsilon_{\mathrm{w}}=0.85$ with acceptable dynamic behavoir is selected. The final spring was designed in an FEM calculation so that the mean square error of the spring curve to the selected one is less than $3 \%$.

\section{References}

[1] Christoph Holtmann, "Design and optimization method for a high power eddy current brake with a magneto isotropic material structure for the use in electrified heavy duty trucks," in EUROBRAKE 2021.

[2] — , "Bremsvorrichtung und fahrzeug," Patent DE 102018 212386 B4, 25.07.2018.

[3] S. E. Gay and M. Ehsani, "Optimized design of an integrated eddy-current and friction brake for automotive applications," in 2005 IEEE Vehicle Power and Propulsion Conference. IEEE, 07-09 Sept. 2005, pp. 189-193.

[4] S. Huang, J. Bao, S. Ge, Y. Yin, and T. Liu, "Design of a frictional-electromagnetic compound disk brake for automotives," Proceedings of the Institution of Mechanical Engineers, Part D: Journal of Automobile Engineering, vol. 234, no. 4, pp. 1113-1122, 2020.

[5] K. Wang, R. He, J. Tang, and R. Liu, "Design and thermal analysis of a novel permanent magnet-friction integrated brake for vehicle," Thermal Science, vol. 24, no. 3 Part A, pp. 1827-1834, 2020

[6] E.-C. von Glasner, "Bremssysteme und bremsverhalten von nutzfahrzeugen und zügen," in Bremsenhandbuch. Springer, 2012, pp. 165-197.

[7] C. Holtmann and A. Mockel, "2d reluctance model of an eddy current brake with a magneto isotropic material structure," in 2020 International Conference on Electrical Machines (ICEM). IEEE, 23.08.2020 - 26.08.2020, pp. 578-584.

[8] O. B. Mawardi, "On the concept of coenergy," Journal of the Franklin Institute, vol. 264, no. 4, pp. 313-332, 1957.

[9] D. Jeltsema and J. M. A. Scherpen, "Multidomain modeling of nonlinear networks and systems," IEEE Control Systems Magazine, vol. 29, no. 4, pp. 28-59, 2009.

[10] M. Hadžiselimović, P. Virtič, G. Štumberger, T. Marčič, and B. Stumberger, "Determining force characteristics of an electromagnetic brake using co-energy," Journal of magnetism and magnetic materials, vol. 320, no. 20, pp. e556-e561, 2008.

[11] D. Coblaş, D. Broboana, C. Balan, and M. Hejjam, "Numerical simulation of constant velocity squeeze flow," UPB Bul., Series D, vol. 75, no. 2, 2013. 